\title{
ITERATIVE APPROXIMATION OF ANALYTIC EIGENVALUES OF A PARAHERMITIAN MATRIX EVD
}

\author{
Stephan Weiss ${ }^{1}$, Ian K. Proudler ${ }^{1}$, Fraser K. Coutts ${ }^{1}$, and Jennifer Pestana ${ }^{2}$ \\ ${ }^{1}$ Dept. of Electronic \& Electrical Eng., University of Strathclyde, Glasgow, Scotland \\ ${ }^{2}$ Dept. of Mathematics \& Statistics, University of Strathclyde, Glasgow, Scotland
}

\begin{abstract}
We present an algorithm that extracts analytic eigenvalues from a parahermitian matrix. Operating in the discrete Fourier transform domain, an inner iteration re-establishes the lost association between bins via a maximum likelihood sequence detection driven by a smoothness criterion. An outer iteration continues until a desired accuracy for the approximation of the extracted eigenvalues has been achieved. The approach is compared to existing algorithms.
\end{abstract}

\section{INTRODUCTION}

A number of recent broadband array problems such as beamforming [1,2], angle of arrival estimation [3,4], blind source separation [5], multichannel coding [6,7], or MIMO system design [8-11] have been successfully formulated and solved using polynomial matrix algebra. Central to this has been the McWhirter decomposition [12], which factorises a parahermitian matrix $\boldsymbol{R}(z)$, i.e. a matrix that is equal to its parahermitian conjugate $\boldsymbol{R}^{\mathrm{P}}(z)=\boldsymbol{R}^{\mathrm{H}}\left(1 / z^{*}\right)=\boldsymbol{R}(z)$. Typically a parahermitian matrix arises as a cross-spectral density (CSD) matrix, i.e. the $z$-transform of a space-time covariance matrix. The factorisation results in

$$
\boldsymbol{R}(z) \approx \boldsymbol{U}(z) \boldsymbol{D}(z) \boldsymbol{U}^{\mathrm{P}}(z)
$$

where $\boldsymbol{U}(z)$ is paraunitary, i.e. $\boldsymbol{U}(z) \boldsymbol{U}^{\mathrm{P}}(z)=\boldsymbol{U}^{\mathrm{P}}(z) \boldsymbol{U}(z)=$ I. The parahermitian matrix $\boldsymbol{D}(z)=\operatorname{diag}\left\{d_{1}(z), \ldots, d_{M}(z)\right\}$ is spectrally majorised, i.e.

$$
d_{m}\left(\mathrm{e}^{\mathrm{j} \Omega}\right) \geq d_{m+1}\left(\mathrm{e}^{\mathrm{j} \Omega}\right), \quad \forall \Omega \in \mathbb{R}, m=1 \ldots(M-1) .
$$

Several algorithms exist that are proven to converge to a diagonalised $\boldsymbol{D}(z)$, including the sequential best rotation (SBR2, [7, 12, 13]) and sequential matrix diagonalisation (SMD, [14-16]) families of algorithms. In general, spectral majorisation is encouraged; SBR2 has even been shown to converge to this solution [17].

This work was supported in parts by the Engineering and Physical Sciences Research Council (EPSRC) Grant number EP/S000631/1 and the MOD University Defence Research Collaboration in Signal Processing.
In $[18,19]$, we have shown that for an analytic parahermitian $\boldsymbol{R}(z)$, a parahermitian matrix EVD (PhEVD)

$$
\boldsymbol{R}(z)=\boldsymbol{Q}(z) \boldsymbol{\Lambda}(z) \boldsymbol{Q}^{\mathrm{P}}(z)
$$

exists with an analytic paraunitary $\boldsymbol{Q}(z)$ and an analytic diagonal $\boldsymbol{\Lambda}(z)$, unless $\boldsymbol{R}(z)$ emerges from multiplexed data [19]. The spectrally majorised solution arises from a permutation of the analytic solution, as shown in the example in Fig. 1. As a consequence, the factors in the McWhirter decomposition in (1) may have to approximate non-differentiable functions in case of the eigenvalues, and discontinuous functions in case of the eigenvectors [18]. Therefore, much higher approximation orders are required to model the factors in (1) compared to those in (3).

We are therefore interested in algorithms that can approximate the analytic solution in (3). The only currently existing attempt is by Tohidian et al. [20], who have chosen a DFTbased approach over SBR2 and SMD algorithms, which operate in the time domain. The approach in [20] does not specify how the DFT length is chosen, and drives the analyticity of the solution by a condition on the eigenvectors. Below, in Sec. 2, we summarise the main results of $[18,19]$, and present the challenges of a DFT-based approach in Sec. 3: by losing the coherence between frequency bins, an association of eigenvalues across bins must be established. We argue why the reliance on the eigenvectors for an analytic solution in [20] can be misleading, and therefore concentrate exclusively on the eigenvalues. For the association, we derive a metric for an optimum solution, which then drives an inner maximum likelihood search in Sec. 4. An outer, iterative scheme is outlined and proven to converge in Sec. 5. Finally, simulation results are presented in Sec. 6 and conclusions are drawn in Sec. 7.

\section{PARAHERMITIAN MATRIX EVD}

For the PhEVD in (3) with eigenvalues in the diagonal parahermitian $\boldsymbol{\Lambda}(z)$ and the eigenvectors as the columns of $\boldsymbol{U}(z)$, we can state:

Theorem 1 (Existence and uniqueness.) Let $\boldsymbol{R}(z): \mathbb{C} \rightarrow$ $\mathbb{C}^{M \times M}$ be a parahermitian matrix that is analytic in $z$ in at least an annulus containing the unit circle, that cannot 

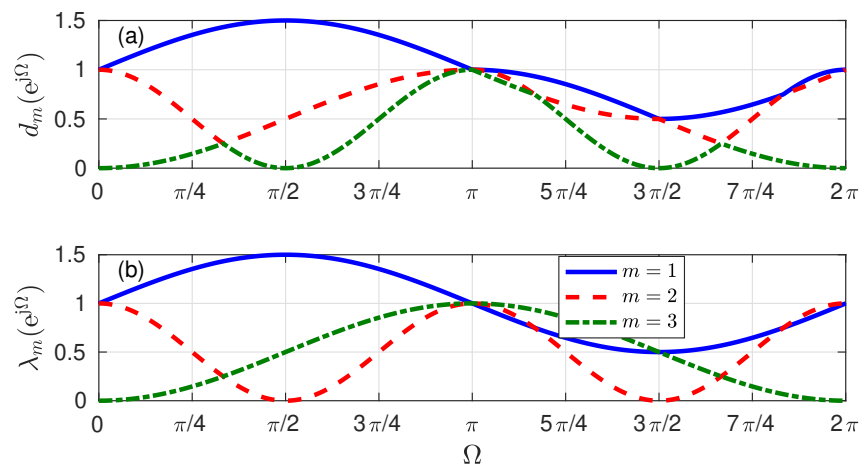

Fig. 1. (a) spectrally majorised and (b) analytic eigenvalues of a matrix $\boldsymbol{R}(z)$, evaluated on the unit circle, $z=\mathrm{e}^{\mathrm{j} \Omega}$.

be related to a block-circulant matrix by a suitable paraunitary similarity transform. Then there exists a PhEVD according to (3) with unique analytic eigenvalues and analytic eigenspaces, within which analytic eigenvectors can be determined up to an arbitrary phase response.

Proof. See $[18,19]$.

The factors $\boldsymbol{\Lambda}(z)$ and $\boldsymbol{U}(z)$ are generally transcendental. Using a Laurent polynomial approximation of order $N$ for the factors $\hat{\boldsymbol{Q}}^{(N)}(z)$ and $\hat{\boldsymbol{\Lambda}}^{(N)}(z)$ we can state:

Theorem 2 (Polynomial approximation.) The best Nthorder approximation $\hat{\boldsymbol{\Lambda}}^{(N)}(z)$ of an analytic $\boldsymbol{\Lambda}(z)$ in the least squares sense is obtained by truncating $\Lambda(z)$ to the required order.

Proof. We consider one eigenvalue $\lambda(z)$ in $\boldsymbol{\Lambda}(z)$. Because of its analyticity, we can write

$$
\lambda(z)=\sum_{n=-\infty}^{\infty} c_{n} z^{-n},
$$

with coefficients $c_{n} \in \mathbb{C}$. For its approximation $\hat{\lambda}^{(N)}(z)$, we use the Laurent polynomial of even order $N$

$$
\hat{\lambda}^{(N)}(z)=\sum_{n=-N / 2}^{N / 2} \hat{c}_{n} z^{-n} .
$$

For the least squares approximation error, the evaluation on the unit circle, $z=\mathrm{e}^{\mathrm{j} \Omega}$, yields

$$
\begin{aligned}
\xi & =\frac{1}{2 \pi} \int_{-\pi}^{\pi}\left|\lambda\left(\mathrm{e}^{\mathrm{j} \Omega}\right)-\hat{\lambda}^{(N)}\left(\mathrm{e}^{\mathrm{j} \Omega}\right)\right|^{2} \mathrm{~d} \Omega \\
& =\sum_{n=-N / 2}^{N / 2}\left|c_{n}-\hat{c}_{n}\right|^{2}+2 \sum_{n=N / 2+1}^{\infty}\left|c_{n}\right|^{2},
\end{aligned}
$$

where we have exploited $\frac{1}{2 \pi} \int_{-\pi}^{\pi} \mathrm{e}^{\mathrm{j} \Omega n} \mathrm{~d} \Omega=\delta(n), \forall n \in \mathbb{Z}$, and the parahermitian property of $\lambda(z)$, s.t. $c_{-n}=c_{n}^{*}$. Hence,

$$
\min \xi \quad \longleftrightarrow \quad \hat{c}_{n}=c_{n} \forall|n| \leq \frac{N}{2},
$$

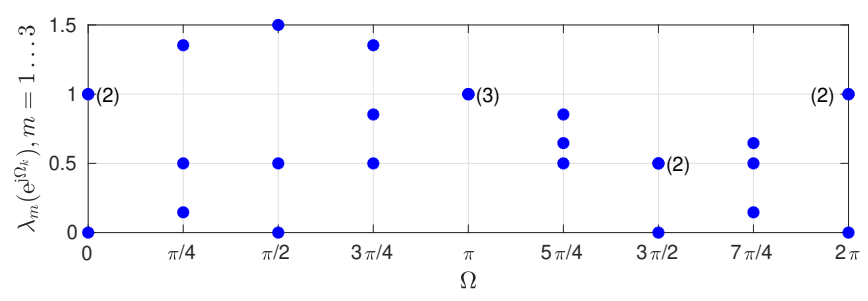

Fig. 2. Eigenvalues $\lambda_{m}\left(\mathrm{e}^{\mathrm{j} \Omega_{\ell}}\right), \ell=0 \ldots(L-1)$ over $L=8$ DFT bins; algebraic multiplicities are indicated in brackets.

i.e. $\hat{\lambda}^{(N)}(z)$ is indeed a truncation of $\lambda(z)$.

\section{DISCRETE APPROXIMATION ON THE UNIT CIRCLE}

\subsection{Evaluation on the Unit Circle}

We evaluate $\boldsymbol{R}(z)$ on the unit circle, $z=\mathrm{e}^{\mathrm{j} \Omega}$, for a number of discrete, equispaced frequency bins $\Omega_{\ell}=\frac{2 \pi}{L} \ell, \ell=0 \ldots(L-$ $1)$, i.e. the set of matrices $\mathbf{R}_{\ell}=\boldsymbol{R}\left(\mathrm{e}^{\mathrm{j} \Omega_{\ell}}\right)$ is obtained by an $L$ point DFT from $\mathbf{R}[\tau]$. In every bin, an EVD

$$
\mathbf{R}_{\ell}=\mathbf{Q}_{\ell} \boldsymbol{\Lambda}_{\ell} \mathbf{Q}_{\ell}^{\mathrm{H}}
$$

is calculated. Since in the DFT domain we have lost coherence between frequency bins, the question that now arises is how to associate eigenvalues across successive bins, i.e. from $\boldsymbol{\Lambda}_{\ell-1}$ to $\boldsymbol{\Lambda}_{\ell}, \ell=1 \ldots(L-1)$.

Example. For the example of $\boldsymbol{R}(z)$ in Fig. 1, an 8-point DFT followed by a bin-wise EVD yields the eigenvalues $\lambda_{m, \ell}, m=1,2,3, \ell=0 \ldots 7$ displayed in Fig. 2 .

The challenge therefore is to assign the set of discrete eigenvalues $\left\{\lambda_{m, \ell}, m=1 \ldots M, \ell=0 \ldots(L-1)\right\}$ such that the $M$ extracted associations $\boldsymbol{\lambda}_{m} \in \mathbb{C}^{L}$ are samples of the desired analytic (and therefore by implication unique) solution. In [20], the association is driven by the eigenvectors, which, if permuted, will display a discontinuity that can be easily detected. However, since in and near $J$-fold algebraic multiplicities the eigenvectors are ill-defined within a $J$-dimensional subspace [21], we here choose to rely entirely on the eigenvalues.

\subsection{Smoothness Metric}

Since analytic functions are infinitely differentiable, we aim to measure the power in the derivatives of the continuous $\lambda_{m}\left(\mathrm{e}^{\mathrm{j} \Omega}\right)$ represented by their discrete samples in $\boldsymbol{\lambda}_{m}, m=$ $1 \ldots M$. Analogous to [22-24], the power in the $p$ th derivative of all $\lambda_{m}\left(\mathrm{e}^{\mathrm{j} \Omega}\right), m=1 \ldots M$ can be determined as

$$
\chi_{p}=\sum_{m} \boldsymbol{\lambda}_{m}^{\mathrm{H}} \mathbf{C}_{p} \boldsymbol{\lambda}_{m},
$$

where $\mathbf{C}_{p}=\mathbf{T D T} \mathbf{T}^{\mathrm{H}}$, with $\mathbf{T}$ the $L$-point DFT matrix and $\mathbf{D}=\operatorname{diag}\left\{\ldots, 2^{2 p}, 1^{2 p}, 0^{2 p}, 1^{2 p}, 2^{2 p}, \ldots\right\}$. 


\subsection{Optimum Solution}

Based on $L$ frequency bins, the smoothest association across these bins therefore will satisfy

$$
\min \sum_{m=1}^{M} \boldsymbol{\lambda}_{m}^{\mathrm{H}} \mathbf{C} \boldsymbol{\lambda}_{m},
$$

where $\mathbf{C}$ is a smoothness matrix which measures a particular derivative power (i.e. $C=C_{p}$ ), or the cumulative power across derivatives via $\mathbf{C}=\sum_{p=1}^{P} \mathbf{C}_{p}$ up to the $P$ th derivative. To check all combinations across $M$ channels and $L$ DFT bins requires the evaluation of $(M !)^{L-1}$ possibilities, which is unrealistic for even moderate values of $M$ and $L$.

\section{MAXIMUM LIKELIHOOD ESTIMATION FOR SMOOTHEST DISCRETE APPROXIMATION}

In order to create a tractable problem, the global search for the minimisation of (10) is replaced by an iterative, binwise, Viterbi-type maximum likelihood sequence estimation approach. The coefficient vectors are grown iteratively: at the $\ell$ th iteration, a new element $\lambda_{\mu, \ell}$ is attached such that $\boldsymbol{\lambda}_{m, i}^{(\ell)} \in \mathbb{R}^{\ell+1}$. The process initialises with 1-d vectors $\boldsymbol{\lambda}_{m, i}^{(0)}=\lambda_{m, 0}$. The subscript $i$ is a path index, and in total $I$ paths are retained at every iteration.

The smoothness of a function associated with a particular path that has the coefficient association in $\boldsymbol{\lambda}_{m, i}^{(\ell)}$ at the $\ell$ iteration, cannot be done via (10) since $\boldsymbol{\lambda}_{m, i}^{(\ell)}$ only has $(\ell+1)$ coefficients. However the vector can be extended to dimension $L$ by free variables, such that a resulting $\lambda_{m}\left(\mathrm{e}^{\mathrm{j} \Omega}\right)$ would be maximally smooth. This can be achieved by partitioning C such that

$$
\mathbf{C}=\left[\begin{array}{ll}
\mathbf{C}_{1} & \mathbf{C}_{2} \\
\mathbf{C}_{2}^{\mathrm{H}} & \mathbf{C}_{4}
\end{array}\right]
$$

with $\mathbf{C}_{1} \in \mathbb{R}^{(\ell+1) \times(\ell+1)}$ and all other matrix dimensions accordingly. Formulating the Schur complement

$$
\mathbf{C}_{\mathrm{r}}=\mathbf{C}_{1}-\mathbf{C}_{2} \mathbf{C}_{4}^{-1} \mathbf{C}_{2}^{\mathrm{H}},
$$

the smoothness of the $i$ th path up to bin $\ell$ can be calculated as

$$
\chi_{i}=\sum_{m} \boldsymbol{\lambda}_{m, i}^{(\ell), \mathrm{H}} \mathbf{C}_{\mathrm{r}} \boldsymbol{\lambda}_{m, i}^{(\ell)} .
$$

Since $\mathbf{C}$ is rank deficient by construction, the inversion in (12) generally requires regularisation. At the end of the $\ell$ th iteration, the $I$ paths with the smallest metric $\chi_{i}$ in (13) are retained. If any of the remaining paths has a value $\chi_{i}$ that exceeds that of the spectrally majorised solution, it can also be pruned.

Once this algorithm has reached the last bin $\ell=(L-1)$, the path with the lowest metric (and therefore highest smoothness of the associated function) is retained. In that case, let the surviving coefficients be recorded in vectors $\boldsymbol{\lambda}_{m}^{(L)}, m=$ $1 \ldots M$.

\section{ITERATIVE ORDER INCREASE AND CONVERGENCE}

In an outer optimisation loop, we exploit analyticity of the extracted eigenvalues to drive the estimation of a suitable DFT order. We start by setting $L_{0}=2^{\left\lceil\log _{2}(N+1)\right\rceil}$ as the initial DFT length, with $N$ the order of $\mathbf{R}(z)$ and $\lceil\cdot\rceil$ the ceiling operator, and evaluate (8) to obtain $\boldsymbol{\lambda}_{m}^{\left(L_{0}\right)}, m=1 \ldots M$.

At the $k$ th iteration, the DFT length is doubled to $L_{k}=$ $2^{k} L_{0}$, and the maximum likelihood sequence approach of Sec. 4 returns $\boldsymbol{\lambda}_{m}^{\left(L_{k}\right)}, m=1 \ldots M$. Note that in going from the $(k-1)$ st to the $k$ th iteration, the EVDs in half of the bins have already been calculated. According to Theorem 2, in the time domain, the $N$ coefficients of a least squares approximation should match the first $N$ coefficients of the analytic solution. However in the DFT domain, the multiplication on the r.h.s. of (8) corresponds to a cyclic convolution in the time domain [25], resulting in wrap-around. This also impacts on the assignment of the bins values $\lambda_{m}^{\left(L_{k}\right)}$. As a coarse criterion on whether the DFT is sufficiently long, we can therefore compare the assignment of bins between subsequent iterations. The metric

$$
E_{1}=\sum_{m}\left\|\boldsymbol{\lambda}_{m}^{\left(L_{k-1}\right)}-\mathbf{A} \boldsymbol{\lambda}_{m}^{\left(L_{k}\right)}\right\|^{2},
$$

uses $\mathbf{A}=\mathbf{I}_{L_{k-1}} \otimes\left[\begin{array}{ll}1 & 0\end{array}\right] \in \mathbb{Z}^{L_{k-1} \times L_{k}}$, with $\otimes$ denoting the Kronecker product, to perform a decimation by two. The quantity $E_{1}$ in (14) will be zero if the bin assigments are identical or permutations only affect eigenvalues at an algebraic multiplicity, or a small value if very closely spaced eigenvalues are permuted. If $E_{1}$ exceeds a threshold, then wraparound is deemed severe enough to merit a $(k+1)$ st iteration.

Once $E_{1}$ falls below a desired threshold, the accuracy of the approximation is assessed in further detail by considering the time domain coefficients. As the DFT order increases, the wrap-around error diminishes, and the difference between the time domain cofficients at iterations $k$ and $(k-1)$ should become smaller. Based on $\mathbf{T}_{L_{k}}$ being an $L_{k}$-point DFT matrix, a measure for the approximation error in the time domain therefore is

$$
\begin{aligned}
E_{2} & =\sum_{m} \| \mathbf{T}_{L_{k-1}}^{\mathrm{H}} \boldsymbol{\lambda}_{m}^{\left(L_{k-1}\right)} \\
& -\left[\begin{array}{lll}
\mathbf{I}_{L_{k-1} / 2} & \mathbf{0}_{L_{k-1}} & \mathbf{0}_{L_{k-1} / 2} \\
\mathbf{0}_{L_{k-1} / 2} & \mathbf{I}_{L_{k-1} / 2}
\end{array}\right] \mathbf{T}_{L_{k}}^{\mathrm{H}} \boldsymbol{\lambda}_{m}^{\left(L_{k}\right)} \|_{2}^{2} .
\end{aligned}
$$

The iteration continues until $E_{2}$ falls below a desired threshold $E_{2, \max }$, or until a maximum permissible DFT length $L_{k, \text { max }}$ is reached.

Conjecture 1 (Convergence.) If (10) is globally minimised, then the overall algorithm as described above is guaranteed to converge. 

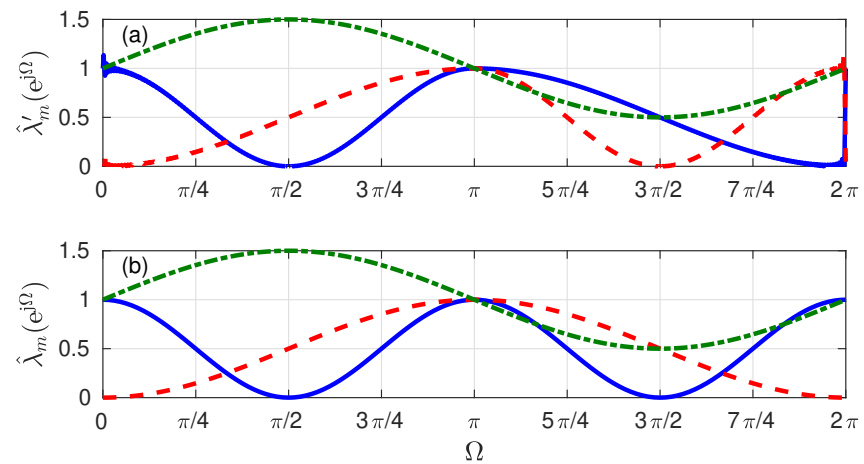

Fig. 3. Approximated analytic eigenvalues using (a) Tohidian et al. [20] and (b) the proposed approach.

Justification. Once the DFT length is sufficient to reduce wrap-around below some level, a stable association of bin values is achieved. Since there is only one analytic solution for the eigenvalues, this is where a stable association has to settle. Therefore, the wrap-around is caused by truncated coefficients, the truncation and therefore approximation error will decrease with the increasing DFT length. Thus, $E_{2}$ also provides a measure for the approximation error defined in (6).

\section{SIMULATIONS AND RESULTS}

We first focus on the example of an individual CSD matrix $\boldsymbol{R}(z)$, that possesses the ground truth analytic eigenvalues

$$
\begin{aligned}
& \lambda_{1}(z)=-j \frac{1}{4} z+1+j \frac{1}{4} z^{-1} \\
& \lambda_{2}(z)=\frac{1}{4} z^{2}+\frac{1}{2}+\frac{1}{4} z^{-2} \\
& \lambda_{3}(z)=-\frac{1}{4} z+\frac{1}{2}-\frac{1}{4} z^{-1}
\end{aligned}
$$

as characterised in Fig. 1(b). While the approach in [20] often succeeds in extracting analytic eigenvalues if the DFT length is selected sufficiently long, this is not guaranteed to occur [26]. The association between bins is driven by the examination of the eigenvectors, and for the case in Fig. 3(a) the algebraic multiplicity at $\Omega=\pi$ misleads the selection, which results in two estimated eigenvalues $\hat{\lambda}_{m}^{\prime}(z), m=2,3$, that approximate discontinuities at $\Omega=2 \pi$. In contrast, the proposed algorithm starts with $L_{0}=32$, terminates with $L_{2}=128$ and an error $E_{2} \approx 10^{-14}$, and extracts the correct analytic estimates $\hat{\lambda}_{m}(z), m=1,2,3$, as shown in Fig. 3(b).

Secondly, the proposed algorithm is tested on an ensemble of matrices $\boldsymbol{R}(z): \mathbb{C} \rightarrow \mathbb{C}^{4 \times 4}$ of order 30 , which are generated by the randomised source model in [14]. In this case, the ground truth eigenvalues are known, and are Laurent polynomials of order 14. Over an ensemble of $10^{5}$ realisations, the analytic eigenvalues are extracted correctly in all cases, typically using two or three outer iterations of the algorithm, and with an error $E_{2}<10^{-13}$.

For a third experiment, an ensemble $\boldsymbol{R}(z)=\boldsymbol{A}^{\mathrm{P}}(z) \boldsymbol{A}(z)$ is based on a matrix $\boldsymbol{A}(z): \mathbb{C} \rightarrow \mathbb{C}^{4 \times 4}$ of order 10, with ele-

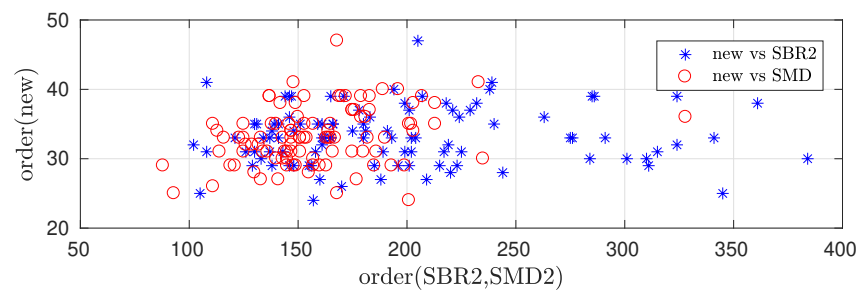

Fig. 4. Order comparison of extracted eigenvalues comparing the new algorithm with SBR2 [12] and SMD [14].

\begin{tabular}{|l||r|r|r|}
\hline algorithm & new & SBR2 [12] & SMD [14] \\
\hline \hline order & $33.2 \pm 4.1$ & $196.3 \pm 62.9$ & $159.4 \pm 33.3$ \\
\hline time $/[\mathrm{s}]$ & $39.7 \pm 1.6$ & $0.2 \pm 1.0$ & $3.5 \pm 0.4$ \\
\hline
\end{tabular}

Table 1. Summary of experiment.

ments drawn from a circularly symmetric uncorrelated Gaussian distribution. In this case, the ground truth eigenvalues are not known, but are guaranteed to be analytic and most likely transcendental, i.e. absolutely convergent but infinite Laurent series. For the decomposition, we compare the proposed ("new") algorithm with a stopping criterion of either $E_{2, \max }=10^{-4}$ or $L_{k, \max }=2^{10}$ to SBR2 [12] and SMD [14] with a similarly set precision, for an ensemble of 100 random realisations of $\boldsymbol{R}(z)$.

The algorithms converge in all cases with the orders of the extracted eigenvalues compared in Fig. 4. In general, both SBR2 and SMD require substantially higher order for the approximation despite internal trimming of the polynomial orders of decomposition factors [12,27-30], since they encourage (SMD) or are guaranteed to yield (SBR2, [17]) spectrally majorised eigenvalues. Some mean and standard deviation values of the experiment are summarised in Tab. 1, where it is evident that the proposed algorithm currently takes significantly more time to compute than the efficiently implemented SBR2 and SMD. However, the strive for analyticity ensures that the proposed method attains much lower order for its extracted eigenvalues.

\section{CONCLUSIONS}

This paper has presented an algorithm for the extraction of analytic eigenvalues, which is based on the eigenvalues only, therefore avoiding problems in the only currently existing DFT-based polynomial matrix EVD algorithm [20]. If an inner optimisation loop succeeds in associating eigenvalues across DFT-bins in a smooth fashion, the outer iteration of the algorithm is conjectured to converge. The approach still requires algorithmic improvements, but provides very promising results in reducing the order of extracted eigenvalues compared to state-of-the-art methods of the SBR2 [7, 12] and SMD [14] algorithm families. 


\section{REFERENCES}

[1] S. Redif, J.G. McWhirter, P.D. Baxter, and T. Cooper, "Robust broadband adaptive beamforming via polynomial eigenvalues," in OCEANS, Boston, MA, Sep. 2006, pp. 1-6.

[2] S. Weiss, S. Bendoukha, A. Alzin, F.K. Coutts, I.K. Proudler, and J.A. Chambers, "MVDR broadband beamforming using polynomial matrix techniques," in European Signal Processing Conference, Nice, France, Sep. 2015, pp. 839-843.

[3] M. Alrmah, S. Weiss, and S. Lambotharan, "An extension of the MUSIC algorithm to broadband scenarios using polynomial eigenvalue decomposition," in European Signal Processing Conference, Barcelona, Spain, Aug. 2011, pp. 629-633.

[4] S. Weiss, M. Alrmah, S. Lambotharan, J.G. McWhirter, and M. Kaveh, "Broadband angle of arrival estimation methods in a polynomial matrix decomposition framework," in IEEE 5th Int. Workshop on Computational Advances in Multi-Sensor Adaptive Processing, St. Martin, Dec. 2013, pp. 109-112.

[5] S. Redif, S. Weiss, and J.G. McWhirter, "Relevance of polynomial matrix decompositions to broadband blind signal separation,” Signal Processing, vol. 134, pp. 76-86, May 2017.

[6] S. Weiss, S. Redif, T. Cooper, C. Liu, P. Baxter, and J.G. McWhirter, "Paraunitary oversampled filter bank design for channel coding," EURASIP Journal on Advances in Signal Processing, vol. 2006, pp. 1-10, 2006.

[7] S. Redif, J.G. McWhirter, and S. Weiss, "Design of FIR paraunitary filter banks for subband coding using a polynomial eigenvalue decomposition," IEEE Transactions on Signal Processing, vol. 59, no. 11, pp. 5253-5264, Nov. 2011.

[8] C.H. Ta and S. Weiss, "A jointly optimal precoder and block decision feedback equaliser design with low redundancy," in European Signal Processing Conference, Poznan, Poland, Sep. 2007, pp. 489-492.

[9] N. Moret, A. Tonello, and S. Weiss, "Mimo precoding for filter bank modulation systems based on psvd," in IEEE 73rd Vehicular Technology Conference, Budapest, Hungary, May 2011, pp. 1-5,

[10] S. Weiss, N. Moret, A.P. Millar, A. Tonello, and R.W. Stewart, "Initial results on an MMSE precoding and equalisation approach to MIMO PLC channels," in IEEE International Symposium on Power Line Communications and Its Applications, Venice, Italy, April 2011, pp. 146-152.

[11] A. Ahrens, A. Sandmann, E. Auer, and S. Lochmann, "Optimal power allocation in zero-forcing assisted PMSVD-based optical MIMO systems," in 2017 Sensor Signal Processing for Defence Conference, Edinburgh, UK, Dec. 2017, pp. 1-5.

[12] J.G. McWhirter, P.D. Baxter, T. Cooper, S. Redif, and J. Foster, "An EVD Algorithm for Para-Hermitian Polynomial Matrices," IEEE Transactions on Signal Processing, vol. 55, no. 5, pp. 2158-2169, May 2007.

[13] Z. Wang, J.G. McWhirter, J. Corr, and S. Weiss, "Multiple shift second order sequential best rotation algorithm for polynomial matrix EVD," in European Signal Processing Conference, Nice, France, Sep. 2015, pp. 844-848.

[14] S. Redif, S. Weiss, and J.G. McWhirter, "Sequential matrix diagonalization algorithms for polynomial EVD of parahermitian matrices," IEEE Transactions on Signal Processing, vol. 63, no. 1, pp. 81-89, Jan. 2015.

[15] J. Corr, K. Thompson, S. Weiss, J.G. McWhirter, S. Redif, and I.K. Proudler, "Multiple shift maximum element sequential matrix diagonalisation for parahermitian matrices," in IEEE SSP, Gold Coast, Australia, June 2014, pp. 312-315.
[16] J. Corr, K. Thompson, S. Weiss, J.G. McWhirter, and I.K. Proudler, "Maximum energy sequential matrix diagonalisation for parahermitian matrices," in 48th Asilomar Conference on Signals, Systems and Computers, Pacific Grove, CA, USA, Nov. 2014, pp. 470-474.

[17] J.G. McWhirter and Z. Wang, "A novel insight to the SBR2 algorithm for diagonalising para-hermitian matrices," in IMA Maths in Signal Processing, Birmingham, UK, Dec. 2016.

[18] S. Weiss, J. Pestana, and I.K. Proudler, "On the existence and uniqueness of the eigenvalue decomposition of a parahermitian matrix," IEEE Transactions on Signal Processing, vol. 66, no. 10, pp. 2659-2672, May 2018.

[19] S. Weiss, J. Pestana, I.K. Proudler, and F.K. Coutts, "Correction to "On the uniqueness and existence of the eigenvalue decomposition of a parahermitian matrix"," IEEE Transactions on Signal Processing, vol. 66, no. 23, pp. 6325-6327, Dec. 2018.

[20] M. Tohidian, H. Amindavar, and A.M. Reza, "A DFT-based approximate eigenvalue and singular value decomposition of polynomial matrices," EURASIP J. Adv. Signal Processing, vol. 2013, no. 1, pp. 1-16, 2013.

[21] G.H. Golub and C.F. Van Loan, Matrix Computations, John Hopkins University Press, Baltimore, Maryland, 3rd edition, 1996.

[22] F.K. Coutts, K. Thompson, J. Pestana, I.K. Proudler, and S. Weiss, "Enforcing eigenvector smoothness for a compact DFT-based polynomial eigenvalue decomposition," in 10th IEEE Sensor Array and Multichannel Signal Processing Workshop, July 2018, pp. 1-5.

[23] S. Weiss and M.D. Macleod, "Maximally smooth Dirichlet interpolation from complete and incomplete sample points on the unit circle," in IEEE International Conference on Acoustics, Speech and Signal Processing, Brighton, UK, May 2019.

[24] S. Weiss, I.K. Proudler, and M.D. Macleod, "Measuring smoothness of real-valued functions defined by sample points on the unit circle," in Sensor Signal Processing for Defence Conference, Brighton, UK, May 2019, submitted.

[25] R.E. Crochiere and L.R. Rabiner, Multirate Digital Signal Processing, Prentice Hall, Englewood Cliffs, NJ, 1983.

[26] F.K. Coutts, K. Thompson, S. Weiss, and I.K. Proudler, "A comparison of iterative and DFT-based polynomial matrix eigenvalue decompositions," in IEEE 7th International Workshop on Computational Advances in Multi-Sensor Adaptive Processing, Curacao, Dec. 2017.

[27] J. Foster, J. G. McWhirter, and J. Chambers, "Limiting the order of polynomial matrices within the SBR2 algorithm," in IMA International Conference on Mathematics in Signal Processing, Cirencester, UK, Dec. 2006.

[28] C.H. Ta and S. Weiss, "Shortening the order of paraunitary matrices in SBR2 algorithm," in 6th International Conference on Information, Communications \& Signal Processing, Singapore, Dec. 2007, pp. 1-5.

[29] J. Corr, K. Thompson, S. Weiss, I.K. Proudler, and J.G. McWhirter, "Row-shift corrected truncation of paraunitary matrices for PEVD algorithms," in European Signal Processing Conference, Nice, France, Aug. 2015, pp. 849-853.

[30] J. Corr, K. Thompson, S. Weiss, I.K. Proudler, and J.G. McWhirter, "Shortening of paraunitary matrices obtained by polynomial eigenvalue decomposition algorithms," in Sensor Signal Processing for Defence, Edinburgh, Scotland, Sep. 2015. 